\title{
The anti-obesity potential of Ilex paraguariensis: results from a meta-analysis
}

\author{
Ângelo Filipe Santos Luís ${ }^{1}$, Fernanda da Conceição Domingues ${ }^{1}$, \\ Luísa Maria Jota Pereira Amaral ${ }^{\circledR 2, *}$
}

\begin{abstract}
${ }^{1}$ Centro de Investigação em Ciências da Saúde (CICS-UBI), Universidade da Beira Interior, Covilhã, Portugal, ${ }^{2}$ Centro de Matemática e Aplicações (CMA-UBI), Universidade da Beira Interior, Covilhã, Portugal
\end{abstract}

\begin{abstract}
The dried leaves of Ilex paraguariensis, also known as yerba mate or chimarrão, are used to produce infusions consumed by over 1 million people worldwide, being a traditional drink in several South American countries, particularly, Argentina, Brazil, Uruguay, and Paraguay. The positive effects of I. paraguariensis in obesity have been described and observed in some clinical trials both in animals and in humans. The main goal of this work was to perform a systematic review, complying with the PRISMA (Preferred Reported Items for Systematic Reviews and Meta-Analysis) recommendations, followed by a meta-analysis and Trial Sequential Analysis (TSA), to summarize the results of the recent clinical trials, clarifying the anti-obesity potential of I. paraguariensis. The search for this systematic review was performed on several electronic databases (Pubmed, Web of Science, Scopus, SciELO and Cochrane Library) in July 2017. A total of 22 clinical trials were considered for the quantitative synthesis of the data, totalizing 726 patients enrolled. In conclusion, this systematic review demonstrated the positive effects of I. paraguariensis consumption on the reduction of body weight, body mass index (BMI), and waist circumference of patients, showing its anti-obesity potential.
\end{abstract}

Keywords: Ilex paraguariensis/systematic review. Meta-analysis. Clinical trials. Anti-obesity.

\section{INTRODUCTION}

The dried leaves of Ilex paraguariensis, also known as yerba mate or chimarrão, are used to produce infusions consumed by over 1 million people worldwide, being a traditional drink in several South American countries, particularly, Argentina, Brazil, Uruguay, and Paraguay (Kim et al., 2015). More recently, the aqueous preparations of I. paraguariensis have also been consumed in North America and Europe (Kim et al., 2015). The dried and minced leaves of I. paraguariensis are used to make brewed tea, which is often used both as a source of caffeine, but also as a therapeutic agent because of its pharmacological properties (Bracesco et al., 2011). The concentration of caffeine varies among the different types of tea (green tea: $130 \mathrm{mg} / \mathrm{L}$; white tea: $140 \mathrm{mg} / \mathrm{L}$; black tea: $89 \mathrm{mg} / \mathrm{L})$ and coffee $(70 \mathrm{mg} / \mathrm{L})$, being the

\footnotetext{
*Correspondence: L. Pereira. Centro de Matemática e Aplicações (CMA-UBI), Universidade da Beira Interior. Rua Marquês d'Ávila e Bolama, 6201-001 Covilhã, Portugal. Tel.: +351 275319 700; Fax: +351 275329 183. E-mail: lpereira@ubi.pt
}

infusion of I. paraguariensis a great source of this compound (40 mg/L) (Rostagno et al., 2011).

The recent interest in I. paraguariensis is linked to its high content of phytochemicals and the peculiarity of its phenolic profile, characterized by the wealth in mono and dicaffeoylquinic acids, known for their biological activities (Cardozo Junior, Morand, 2016). It has also been shown that infusions of I. paraguariensis increase the antioxidant protection of plasma and cholesterol particles against ex vivo copper-mediated lipid peroxidation in humans, together with the improvement in the plasma lipid profile of normolipidemic and healthy dyslipidemic individuals (Boaventura et al., 2012; Morais et al., 2009). Furthermore, the consumption of I. paraguariensis beverages, seems to be related to an improvement of glycemic control on pre-diabetic subjects (Klein et al., 2011). The positive effects of I. paraguariensis in obesity have been observed in some clinical trials both in animals and in humans (Pontilho et al., 2015; Gambero, Ribeiro, 2015; Kim et al., 2012).

Overall, 23.2\% of the world's adult population in 2005 was overweight, with $9.8 \%$ being considered 
obese (Kim et al., 2012). The estimated total number of overweight and obese adults in 2008 was 1500 million and 500 million, respectively (Kim et al., 2012). Moreover, in 2016, more than 1.9 billion adults, 18 years and older, were overweight, being 650 million of these considered obese (WHO, 2018). Additionally, 39\% of adults aged 18 years and over were overweight in 2016, and $13 \%$ were obese (WHO, 2018). Since the prevalence of obesity is a major public health concern, due to all the weight-related diseases that result in significant morbidity and mortality, and reduced quality of life, it is important to search for new strategies to help obese people reducing their body weight (Gambero, Ribeiro, 2015). I. paraguariensis appears as a potential new natural approach to treat obesity, being observed that I. paraguariensis modulates the expression of genes that are changed in the obese state and restores them to normal levels of expression (Gambero, Ribeiro, 2015).

Despite the current research focused on the bioactive properties of I. paraguariensis, which has led to an increase in clinical trials in humans, contradictory results were frequently reported. Taking this context into consideration, the main goal of this work was to perform a systematic review, complying with the PRISMA (Preferred Reported Items for Systematic Reviews and Meta-Analysis) recommendations, followed by a meta-analysis and Trial Sequential Analysis (TSA), to summarize the results of the recent clinical trials, clarifying the anti-obesity potential of I. paraguariensis.

\section{MATERIAL AND METHODS}

\section{Search strategy, inclusion criteria and study selection}

The search for this systematic review was performed on several electronic databases (Pubmed, Web of Science, Scopus, SciELO and Cochrane Library) in July 2017. The databases were queried using the Boolean operator tools, with the following strategy: (Ilex paraguariensis OR yerba mate OR yerba maté OR chimarrão) AND (clinical trial OR trial) AND human. The list of references of the articles reviewed were also checked to find additional studies. Moreover, several academic theses of the authors of some articles were also found (Google Scholar) and were used to complement the gathering of the results. Following the PRISMA recommendations (Moher et al., 2015), titles and abstracts of records retrieved were initially screened and the full texts of those considered relevant were then downloaded and analysed in detail. Two authors independently conducted the literature selection process, with a third being consulted in case of discrepancies. To be included in this systematic review, studies must accomplish the following criteria: to be clinical trials in humans, to present a true control group, to show the results of the outcomes at the baseline and at the end of the trial, and to indicate the standard deviation (SD) of the measurements.

\section{Data extraction and synthesis}

After the selection of the studies, the clinical trials included were carefully analyzed and the following data were extracted and summarized: first author's last name, year of publication, location, study design, number of patients (intervention and control groups), type of patients, mean age and gender of patients, study duration, dosage of I. paraguariensis and the list of outcomes of interest. According to the PRISMA methodology (Moher et al., 2015), two authors independently reviewed and extracted the data using a prespecified protocol. In cases of discordance, a third reviewer was consulted to analyse discrepancies in data extraction.

\section{Risk of bias assessment}

The risk of publication bias of each included study was assessed using the "Cochrane Guide for Review Authors on Assessing Study Quality" which is based on "Cochrane Collaboration's tool for assessing risk of bias" (Ryan et al., 2013). This tool classifies the risk of bias in randomized controlled trials (RCTs) included in reviews as "High risk", "Unclear" or "Low risk" in accordance with 7 domains: random sequence generation, allocation concealment, blinding of participants and personnel, blinding of outcome assessment, incomplete outcome data, selective reporting and other sources of bias (Higgins et al., 2011). This classification was independently assigned by two authors and discrepancies in assessment were resolved through discussions between the authors or by consultation of a third investigator. The results of the risk of bias assessment are presented in a risk of bias summary and in a risk of bias graph, which were sketched using the software Review Manager 5.3 (Version 5.3.5).

\section{Statistical analyses}

For each outcome of interest, an assessment was performed on the pooled effect of the different interventions in terms of weighted mean differences (WMD) between the change from baseline and post-treatment mean values. Data statistical analysis was performed using Comprehensive Meta-Analysis software (Version 2.0) by introducing the number of patients, the differences in mean and respective 
SD values of the outcomes for intervention and control groups. Forest plots were generated to illustrate the studyspecific effect sizes along with a $95 \%$ confidence interval (CI). The random effects model was used, however, when a fixed effects model would be more useful, i.e., when there was little variance in effect sizes, the software automatically converted the random effects model into a fixed effects model (Borenstein, Hedges, Higgins, 2009). The statistic $\mathrm{I}^{2}$ of Higgins was used as a measure of inconsistency across the findings of the included studies. The scale of $\mathrm{I}^{2}$ has a range of 0 to $100 \%$ and values on the order of $25 \%, 50 \%$ and $75 \%$ are considered low, moderate and high heterogeneity, respectively (Higgins et al., 2003). Three different analyses were used to assess the potential impact of publication bias on the present meta-analysis. The first analysis is a funnel plot (Light, Singer, Willett, 1994; Light, Pillemer, 1984). The symmetry of the funnel plots in which mean differences were plotted against their corresponding standard error (SE) was assessed. In the absence of publication bias, the studies were symmetrically distributed in relation to the pooled effect size. Given that the interpretation of a funnel plot is largely subjective, Egger's regression test was performed to test for potential publication bias (Egger et al., 1997). Since this test tends to have lower power, the absence of a significant correlation cannot be taken as evidence of symmetry (Borenstein, Hedges, Higgins, 2009). Therefore, another analysis was used based on the approach of Duval and Tweedie's Trim and Fill (Duval, Tweedie, 2000a; 2000b). Trim and Fill uses an iterative procedure to remove studies that contain a small sample size from the positive (or negative) side of the funnel plot. It then re-computes the effect size at each iteration until the funnel plot is symmetrical in terms of the new effect size, yielding an unbiased estimate of the pooled effect size. This approach allows the best estimate of the unbiased pooled effect size to be obtained and lends itself an intuitive visual display as it creates a funnel plot that includes both the observed studies and the imputed studies.

The sensitivity analysis was also performed by removing each study, one at a time, to evaluate the stability of the results.

Meta-regression analysis was also undertaken to evaluate the association between calculated WMD in body weight, body mass index (BMI) and waist circumference, and the study duration (Borenstein, Hedges, Higgins, 2009).

\section{Trial Sequential Analysis}

TSA is a methodology that uses a combination of techniques in which the evidence required is quantified, providing a value for the required information size (RIS) (Thorlund et al., 2011). The TSA program version 0.9.5.5 Beta (Copenhagen Trial Unit, www.ctu.dk/tsa/downloads/ aspx) was used to adjust the confidence intervals due to sparse data and repeated testing in cumulative meta-analysis. If the cumulative $Z$-curve crosses a trial sequential monitoring boundary or enters the futility area, then it can be concluded that a sufficient level of evidence for the anticipated intervention effect may have been reached and no further trials are needed; whereas if the $Z$-curve does not cross any of the boundaries and the RIS has not been reached, there is insufficient evidence to reach a conclusion. The outcome variables in the present meta-analysis were continuous, so the RIS was estimated based on the empirical data auto-generated by the software per the data input. The TSA was performed at the level of an overall $5 \%$ risk of type I error and a power of $20 \%$.

\section{RESULTS AND DISCUSSION}

\section{Search and selection of studies}

The progression details of the electronic search for this systematic review are represented as a flow-diagram (Figure 1). From the 153 eligible articles, 8 were included in this systematic review with meta-analysis. Among the reasons for exclusion of articles were mostly the lack of a true control group and the absence of SD values for the outcomes of interest. The work of Kim et al. (2012) was divided into 2 different trials because the authors used two durations for the intervention (3 and 6-weeks). A similar procedure was undertaken in the trial of Kim et al. (2015) (6 and 12-weeks). The study of Klein et al. (2011) was divided into 6 different trials taking the possible combinations between the study duration (20, 40 and 60 -days) and the type of patients enrolled in the trial (type 2 diabetes and pre-diabetes) into account. The work of Morais et al. (2009) was divided into 8 different trials, considering the different durations of the study $(20,40,60$ and 90-days) along with the treatment of the dyslipidemic patients with or without statin. As a result, a total of 22 clinical trials were considered for the quantitative synthesis of the data, totalizing 726 patients enrolled.

\section{Included studies and trials characteristics}

The main characteristics of the included trials are summarized in the Table I. The studies cover almost a decade, from 2007 to 2016 . The included trials were mostly performed in Brazil and in the Republic of Korea. It will be important to evaluate the effects of I. paraguariensis 

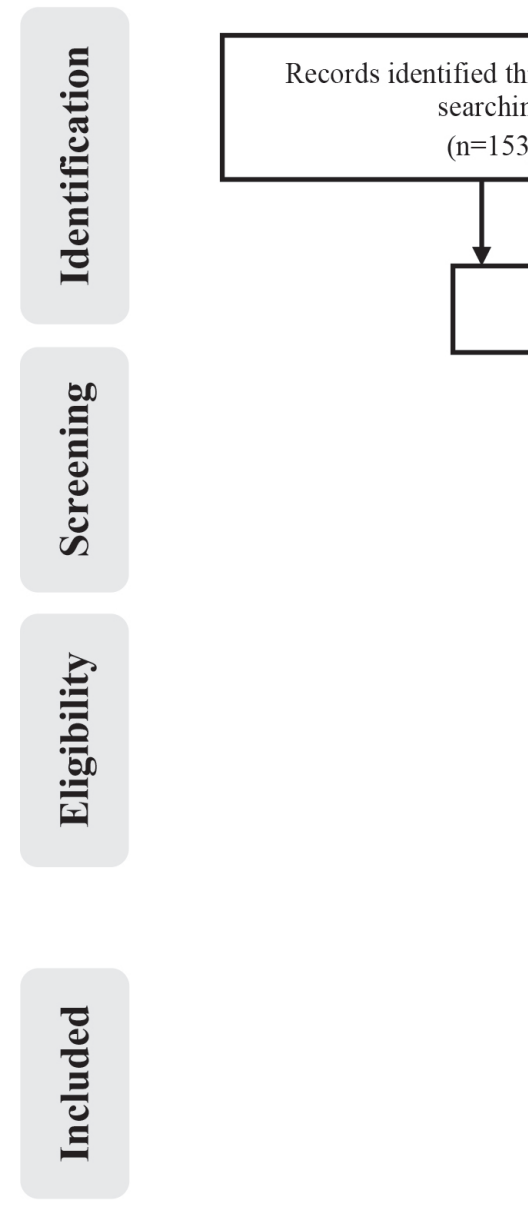

Additional records identified through other sources $(n=31)$

Records after duplicates removed $(\mathrm{n}=85)$

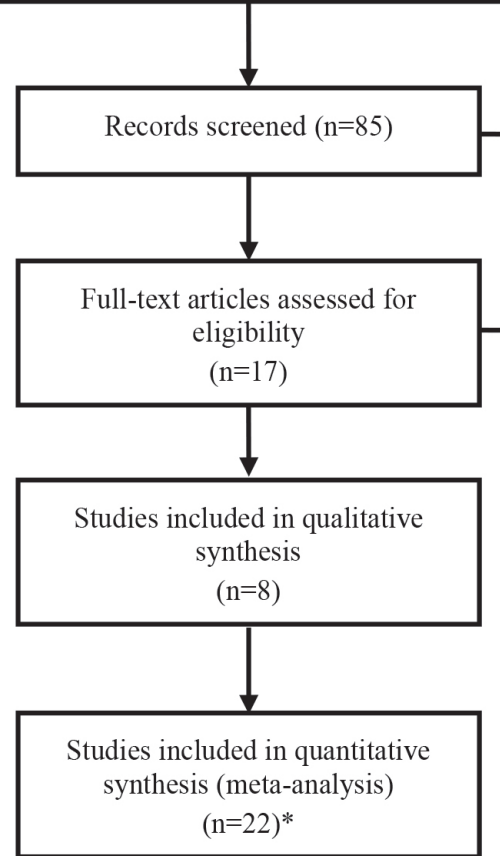

FIGURE 1 - Flow-diagram of database search, trial selection and articles included in this meta-analysis. *The works of Kim et al., 2012 and Kim et al., 2015 were divided into 2 different trials. The work of Klein et al., 2011 was divided into 6 different trials. The work of Morais et al., 2009 was divided into 8 different trials.

consumption on patients from other countries, as several other factors may interfere in the biological effects when consuming polyphenol-rich plants. For example, the bioavailability of the bioactive compounds, and particularly the polyphenols, is extremely affected by their metabolization, which in turn is influenced by the genotype characteristics of a certain region of the world (Rein et al., 2013). The patients subjected to the intervention with I. paraguariensis were mainly dyslipidemic, obese and diabetic. However, it will be necessary to evaluate the effects of I. paraguariensis also in healthy volunteers due to the high concentrations of caffeine found in this plant. I. paraguariensis was administered to the patients mainly by aqueous preparations, varying the duration of the interventions and the dosage.

\section{Risk of publication bias}

The results found in the assessment of the risk of publication bias from the included studies are summarized in Figure 2. The studies of Morais et al. (2009) (from A to H) and Salerno (2007) were nonrandomized and for that reason were not classified to their risk of bias since the Cochrane's tool can only be applied to RCTs. In general, all the trials satisfied the 7 domains of bias defined by Cochrane Collaboration. The included RCTs claimed to be randomized, but only 6 trials detailed the randomization process and so were classified as "Low risk" in the random sequence generation domain. Concerning the performance and detection bias, related to blinding of participants and personnel, and blinding of outcome assessment, respectively, there were 2 studies (Boaventura et al., (2012), and Pontin (2010)) classified as "High risk", since there were doubts regarding the blinding process (single or double). It is important to note, however, that the assessment of risk of publication bias is a subjective task, even when employing the Cochrane's tool, because it is based on the personal judgments of the review authors. 


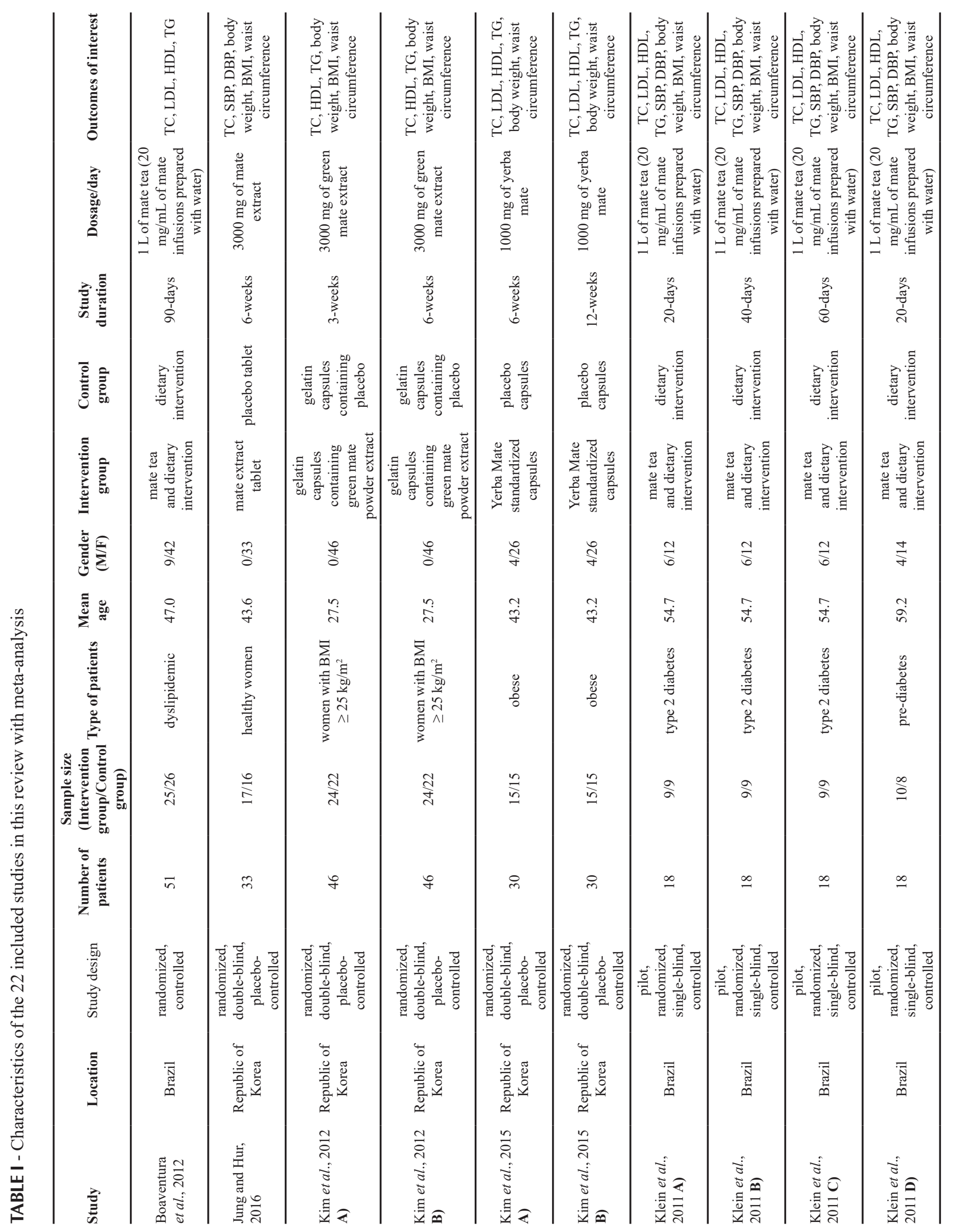




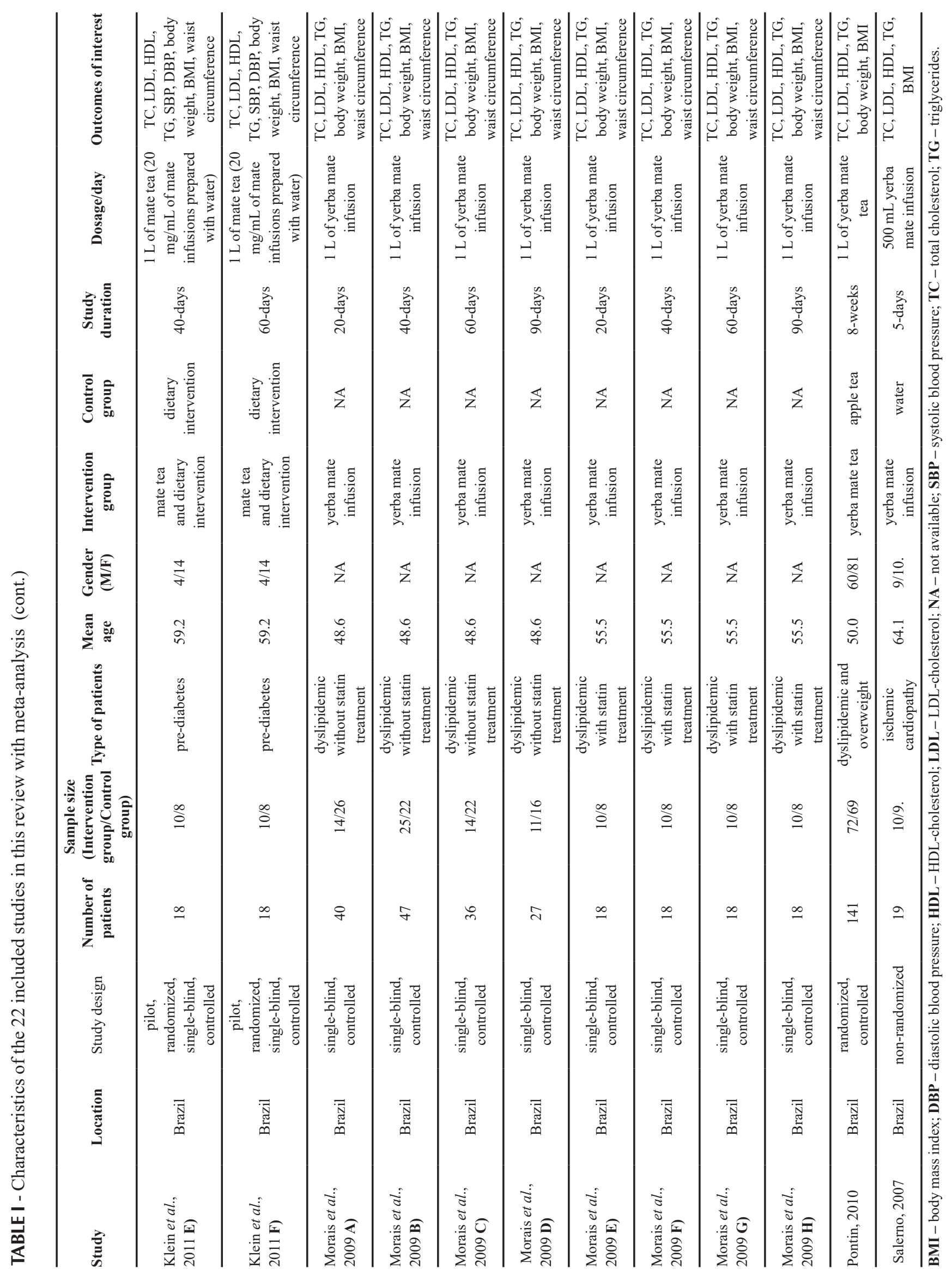




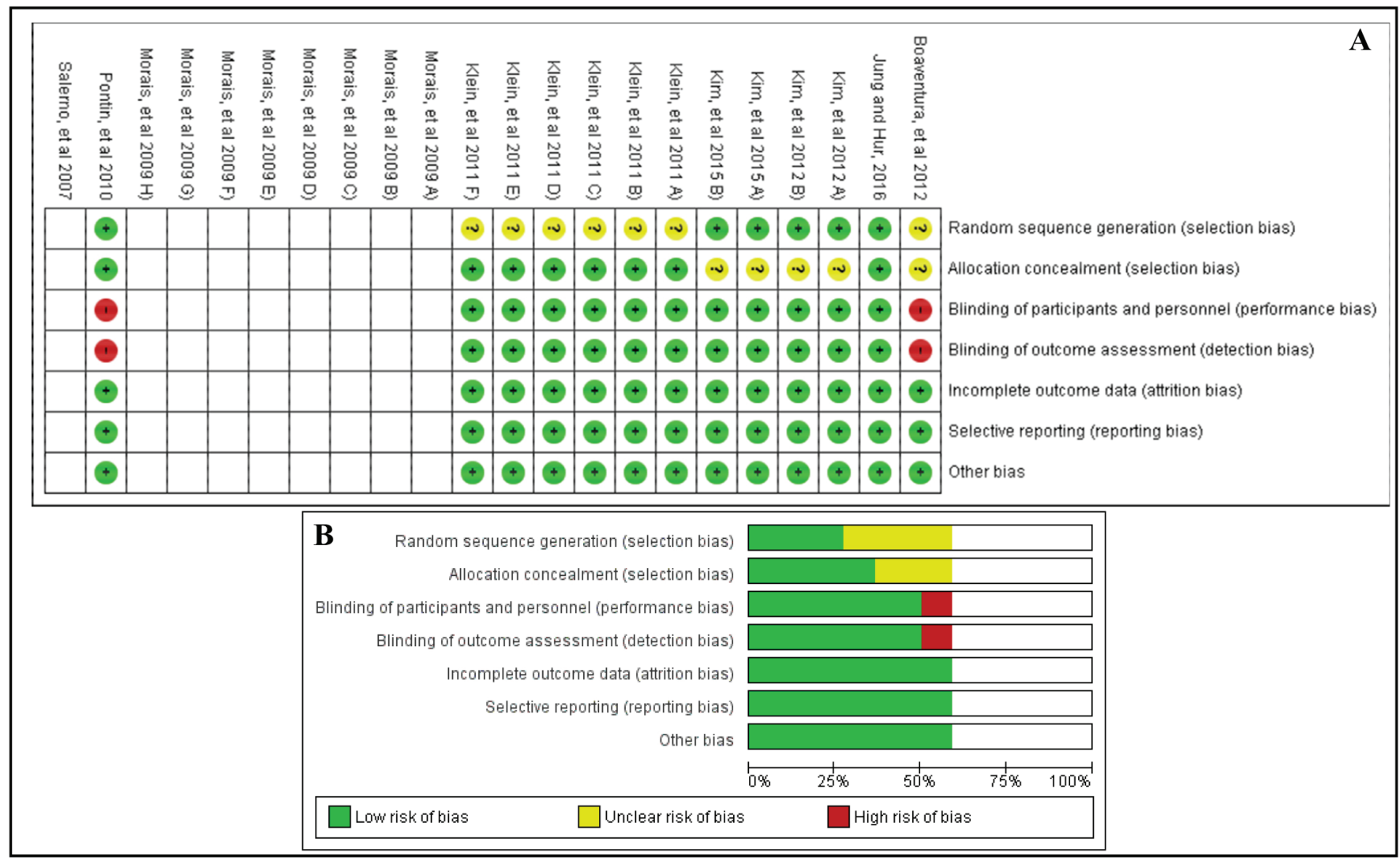

FIGURE 2 - Results of risk of bias assessment regarding the methodological quality of included studies: A) Risk of bias summary: review author's judgments about each risk of bias item for each included study; B) Risk of bias graph: review author's judgments about each risk of bias item presented as percentages across all included studies.

\section{Effects of $I$. paraguariensis consumption on anthropometric measurements, lipid profile and blood pressure}

Several previous reports have suggested that I. paraguariensis may possess anti-obesity properties, together with the potential to ameliorate the blood lipid profile of patients (Boaventura et al., 2012; Gambero, Ribeiro, 2015; Kim et al., 2015). In contrast, I. paraguariensis consumption may cause an increase in blood pressure due to the high concentration in caffeine (Cardozo Junior, Morand, 2016; Pontilho et al., 2015). In this sense, the outcomes considered in this metaanalysis include some anthropometric measurements (body weight, BMI, and waist circumference), lipid profile and blood pressure of the participants in the clinical trials.

The lipid profile comprises the total cholesterol (TC), triglycerides (TG), HDL-cholesterol (HDL) and LDL-cholesterol (LDL). In the case of the blood pressure, the values of systolic blood pressure (SBP) and diastolic blood pressure (DBP) were both considered in the statistical analysis.
Figure 3 represents the forest plots resulting from the meta-analysis for the considered outcomes. Table II summarizes the meta-analysis results. Overall, it is possible to verify that $I$. paraguariensis consumption significantly reduces the body weight (Figure $3 \mathrm{~A}$ ) (WMD: $-0.521 \mathrm{~kg} ; 95 \%$ CI: -0.870 to $-0.172 ; p$-value $=0.003)$ and consequently the BMI (Figure 3B) of patients (WMD: $-0.208 \mathrm{~kg} / \mathrm{m}^{2} ; 95 \% \mathrm{CI}$ : -0.342 to $-0.073 ; p$-value $\left.=0.002\right)$, which together with the significant reduction of waist circumference (Figure 3C) (WMD: $-0.668 \mathrm{~cm}$; 95\% CI: -1.316 to $-0.021 ; p$-value $=0.043)$ demonstrate that, in fact, I. paraguariensis has some potential to treat obesity, which is in agreement with the findings of some clinical trials included in the present meta-analysis. It should be noted that no heterogeneity $\left(\mathrm{I}^{2}=0 \%\right)$ was observed among the trials for these outcomes (Table II). Concerning the results of TSA, it is possible to conclude by the visual inspection of the plots (Figure 4), that, in general, the cumulative $Z$-curve crosses the conventional significance test boundary and RIS-adjusted boundary value, indicating that the evidence needed to reach a conclusion was sufficient and no further trials were needed; thus, additional trials are not required and are unlikely to alter 

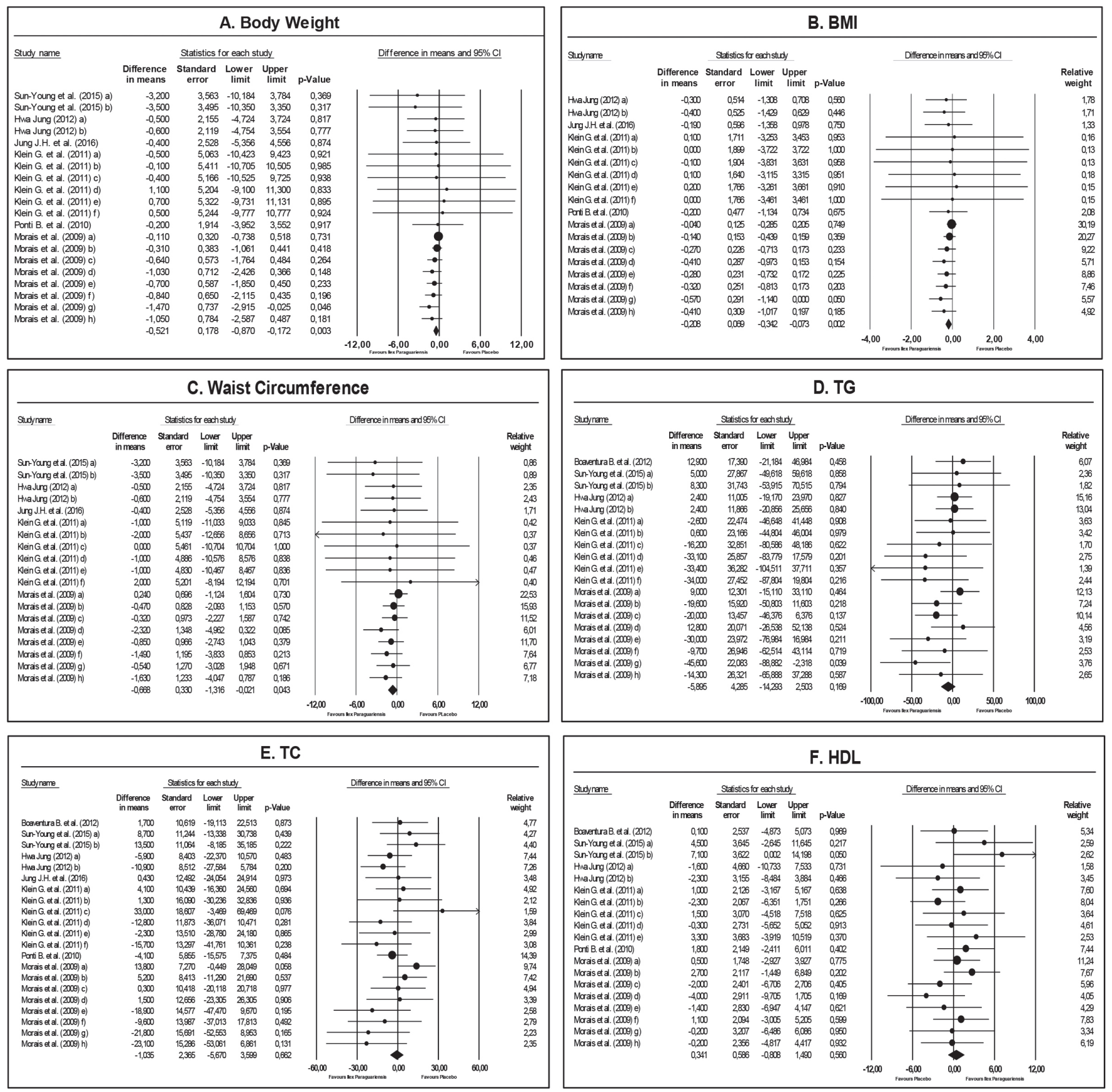

FIGURE 3 - Forest plots of comparisons of the effects I. paraguariensis consumption on the outcomes of this meta-analysis, compared with control arms. BMI - body mass index; DBP - diastolic blood pressure; HDL - HDL-cholesterol; LDL - LDLcholesterol; SBP - systolic blood pressure; TC - total cholesterol; TG - triglycerides.

the conclusions obtained in the present work in what concerns the anthropometric measurements.

Regarding the remaining outcomes under analysis, it was verified that I. paraguariensis have no significant implications in both the lipid profile (Figures 3D, 3E, $3 \mathrm{~F}, 3 \mathrm{G}$ and Table II) and blood pressure of patients (Figures 3H, 3I and Table II). However, the number of clinical trials evaluating the effects of $I$. paraguariensis in these outcomes is reduced, leading to some heterogeneity (Table II) in addition to extremely high values of RIS (Table II), not being reached, indicating that more clinical trials are needed in order to achieve sufficient evidence to reach a conclusion.

Moreover, the TSA program was not able to produce the plots for the remaining outcomes due to the scarcity of data. 

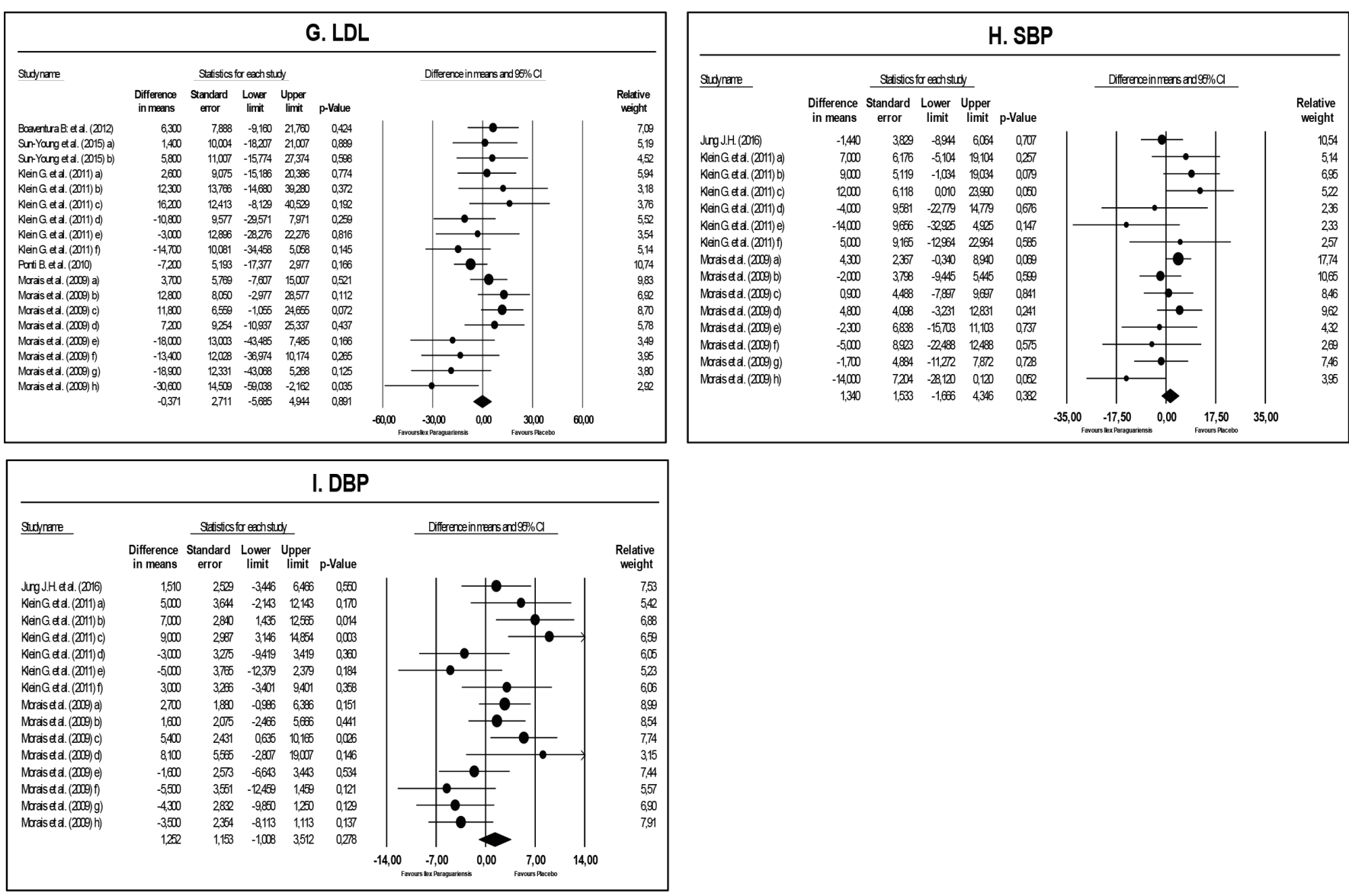

FIGURE 3 (cont.) - Forest plots of comparisons of the effects I. paraguariensis consumption on the outcomes of this meta-analysis, compared with control arms. BMI - body mass index; DBP - diastolic blood pressure; HDL - HDL-cholesterol; LDL - LDLcholesterol; SBP - systolic blood pressure; TC - total cholesterol; TG - triglycerides.

The sensitivity analysis was also performed by excluding one or more studies from the analysis to see how this affected the results. The results showed that the pooled effects of I. paraguariensis consumption on the considered outcomes did not change substantially if a single or a few studies were omitted (data not shown).

Overall, the sensitivity analysis demonstrated that the findings of this meta-analysis on the considered outcomes are robust.

During this systematic review, it was verified that I. paraguariensis was administered to the patients at different intervention durations. Therefore, a metaregression analysis (Figure 5) was conducted to evaluate the association between changes in body weight, BMI and waist circumference, and the study duration. The results of meta-regression suggest no significant correlation between the intervention duration and body weight (Figure 5A; $p$-value $=0.0896), \mathrm{BMI}$ (Figure 5B; $p$-value $=0.105$ ) or waist circumference (Figure 5C; $p$-value $=0.125)$.
In summary, the findings clearly showed that the use of I. paraguariensis might be useful against obesity.

In the previous comprehensive reviews published by Bracesco et al., 2011 and by Gambero and Ribeiro, 2015 , some possible mechanisms of action, regarding the anti-obesity potential of I. paraguariensis, were addressed; however, more studies at cellular and molecular level must be undertaken to better understand this biological activity of I. paraguariensis.

\section{Publication bias}

To analyse the publication bias, funnel plots were generated for the defined outcomes considering the Trim and Fill adjustment (Figure 6). These funnel plots included both the observed studies (shown as blue circles) and the necessary imputed studies (shown as red filled circles) to obtain the absence of bias. It was observed that there is an absence of bias for body weight (Figure 6 A). For the remaining outcomes, some studies are imputed on the 
TABLE II - Meta-analysis results of the effects of I. paraguariensis consumption on the considered outcomes

\begin{tabular}{|c|c|c|c|c|c|c|}
\hline Outcomes & Results & Number of trials & $95 \%$ CI & $p$-value & $I^{2}(\%)$ & RIS \\
\hline & Total & 20 & - & - & - & 579 \\
\hline \multirow[t]{3}{*}{ Body Weight } & WMD observed & - & $-0.521(-0.870 ;-0.172)$ & $0.003 *$ & 0 & \\
\hline & WMD adjusted & - & $-0.521(-0.870 ;-0.170)$ & - & - & \\
\hline & Total & 19 & - & - & - & 499 \\
\hline \multirow[t]{3}{*}{ BMI } & WMD observed & - & $-0.208(-0.342 ;-0.073)$ & $0.002 *$ & 0 & \\
\hline & WMD adjusted & - & $-0.209(-0.343 ;-0.075)$ & - & - & \\
\hline & Total & 19 & - & - & - & 973 \\
\hline \multirow[t]{3}{*}{ Waist Circumference } & WMD observed & - & $-0.668(-1.316 ;-0.021)$ & $0.043 *$ & 0 & \\
\hline & WMD adjusted & - & $-0.504(-1.127 ; 0.119)$ & - & - & \\
\hline & Total & 10 & - & - & - & 2334 \\
\hline \multirow[t]{3}{*}{ TG } & WMD observed & - & $-5.895(-14.293 ; 2.503)$ & 0.169 & 0 & \\
\hline & WMD adjusted & - & $-1.249(-9.540 ; 7.042)$ & - & - & \\
\hline & Total & 21 & - & - & - & 19353 \\
\hline \multirow[t]{3}{*}{$\mathrm{TC}$} & WMD observed & - & $-1.305(-5.670 ; 3.599)$ & 0.662 & 3.869 & \\
\hline & WMD adjusted & - & $-0.001(-4.849 ; 4.829)$ & - & - & \\
\hline & Total & 19 & - & - & - & 64545 \\
\hline \multirow[t]{3}{*}{ HDL } & WMD observed & - & $0.341(-0.808 ; 1.490)$ & 0.560 & 0 & \\
\hline & WMD adjusted & - & $0.341(-0.808 ; 1.490)$ & - & - & \\
\hline & Total & 18 & - & - & - & 1436902 \\
\hline \multirow[t]{3}{*}{ LDL } & WMD observed & - & $-0.371(-5.685 ; 4.944)$ & 0.891 & 33.670 & \\
\hline & WMD adjusted & - & $1.274(-4.369 ; 6.918)$ & - & - & \\
\hline & Total & 15 & - & - & - & 4561 \\
\hline \multirow[t]{3}{*}{ SBP } & WMD observed & - & $1.340(-1.667 ; 4.346)$ & 0.382 & 23.509 & \\
\hline & WMD adjusted & - & $2.621(-0.725 ; 5.966)$ & - & - & \\
\hline & Total & 15 & - & - & - & 2421 \\
\hline \multirow[t]{2}{*}{ DBP } & WMD observed & - & $1.252(-1.008 ; 3.512)$ & 0.278 & 0 & \\
\hline & WMD adjusted & - & $1.252(-1.008 ; 3.512)$ & - & - & \\
\hline
\end{tabular}

BMI - body mass index; CI - confidence interval; DBP - diastolic blood pressure; HDL - HDL-cholesterol; LDL - LDLcholesterol; RIS - required information size; SBP - systolic blood pressure; TC - total cholesterol; TG - triglycerides; WMD - weighted mean differences; * - indicates a significant result.

right or on the left size to "adjust" the funnel plots for the absence of publication bias. Therefore, by observing the funnel plots, it is possible to verify that publication bias cannot be completely excluded. The values of WMD adjusted for the outcomes under analysis are presented in Table II.

In addition to the visual inspection of the funnel plots, the presence of publication bias was explored using Egger's regression test. This test did not indicate evidence of publication bias for the impact of I. paraguariensis consumption on the evaluated outcomes (Table III).

\section{CONCLUSIONS}

In conclusion, this systematic review with metaanalysis and TSA of clinical trials, demonstrated the positive effects of $I$. paraguariensis consumption on the reduction of body weight, BMI, and waist circumference of patients, showing its anti-obesity potential. However, special attention must be given due to the high concentrations of caffeine found in this plant. Future clinical trials should evaluate the effects of 

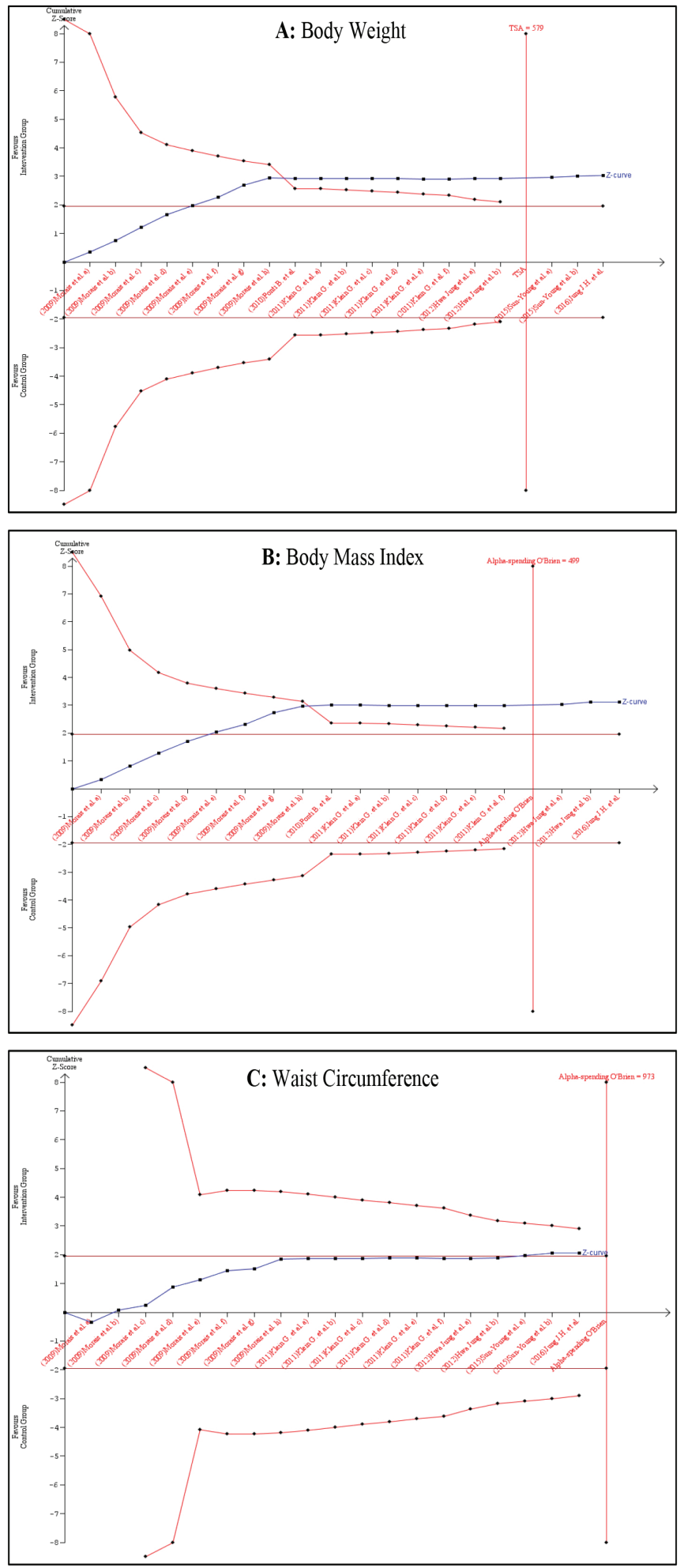

FIGURE 4 - Trial Sequential Analysis (TSA) on pooled results of the effects of I. paraguariensis consumption on the anthropometric measurements considered in this meta-analysis.
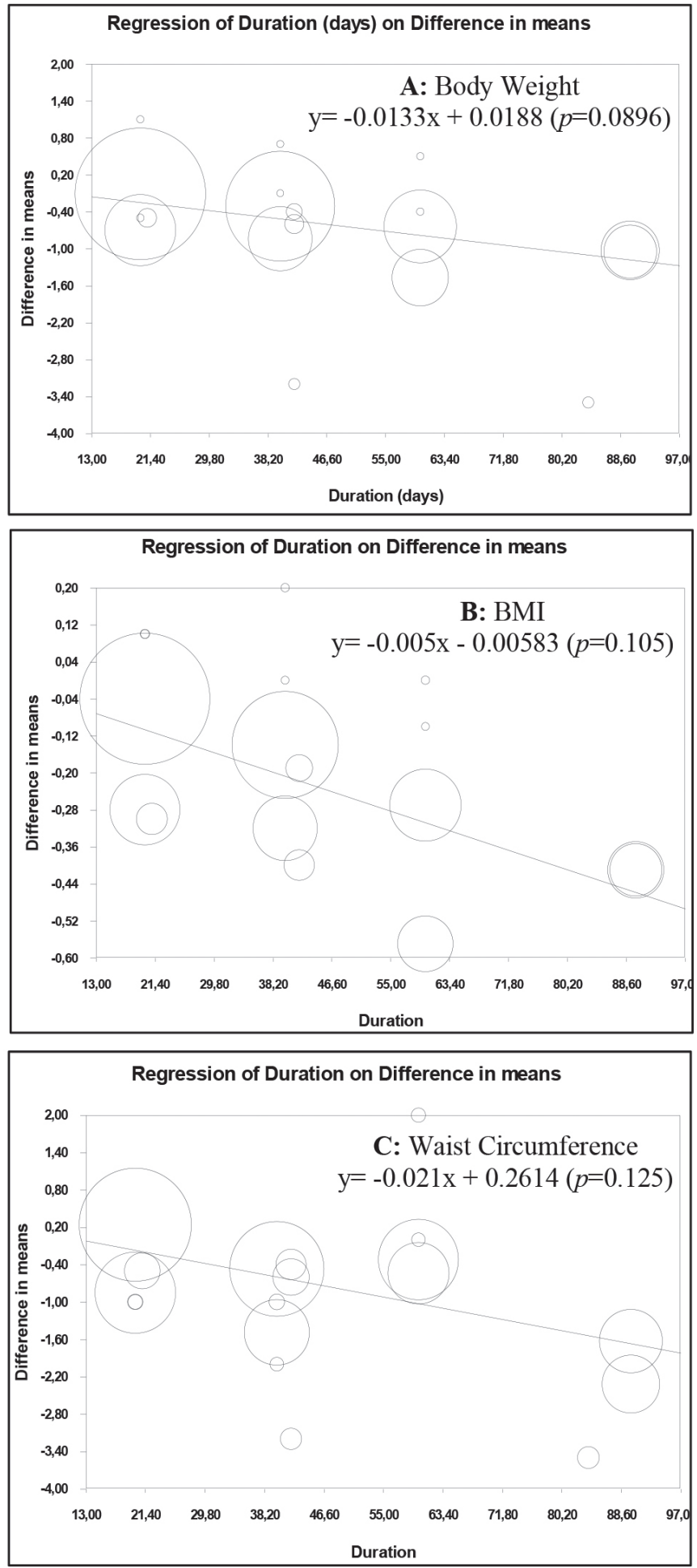

FIGURE 5 - Meta-regression plots of the association between calculated weighted mean differences (WMD) in body weight, body mass index (BMI) and waist circumference, and the study duration. 
TABLE III - Assessment of publication bias for the impact of I. paraguariensis consumption on the considered outcomes

\begin{tabular}{lcccc}
\hline \multirow{2}{*}{ Outcomes } & \multicolumn{4}{c}{ Egger's regression test } \\
\cline { 2 - 5 } & $\mathbf{9 5 \%} \mathbf{C I}$ & $\boldsymbol{t}$ & $\mathbf{d f}$ & $\boldsymbol{p}$-value \\
\hline Body weight & $-0.650 ; 0.130$ & 1.390 & 18 & 0.182 \\
BMI & $-0.655 ; 0.231$ & 1.013 & 16 & 0.326 \\
Waist circumference & $-0.824 ; 0.151$ & 1.455 & 17 & 0.164 \\
TG & $-2.126 ; 0.135$ & 1.857 & 17 & 0.080 \\
TC & $-2.161 ; 1.063$ & 0.712 & 19 & 0.485 \\
HDL & $-1.460 ; 2.233$ & 0.441 & 17 & 0.664 \\
LDL & $-3.015 ; 0.975$ & 1.084 & 16 & 0.295 \\
SBP & $-2.537 ; 0.574$ & 1.360 & 13 & 0.196 \\
DBP & $-3.930 ; 3.630$ & 0.086 & 13 & 0.933 \\
\hline
\end{tabular}

BMI - body mass index; CI - confidence interval; DBP-diastolic blood pressure; $\mathbf{d f}$ - degrees of freedom; HDL-HDL-cholesterol; LDL - LDL-cholesterol; SBP - systolic blood pressure; TC - total cholesterol; TG - triglycerides.
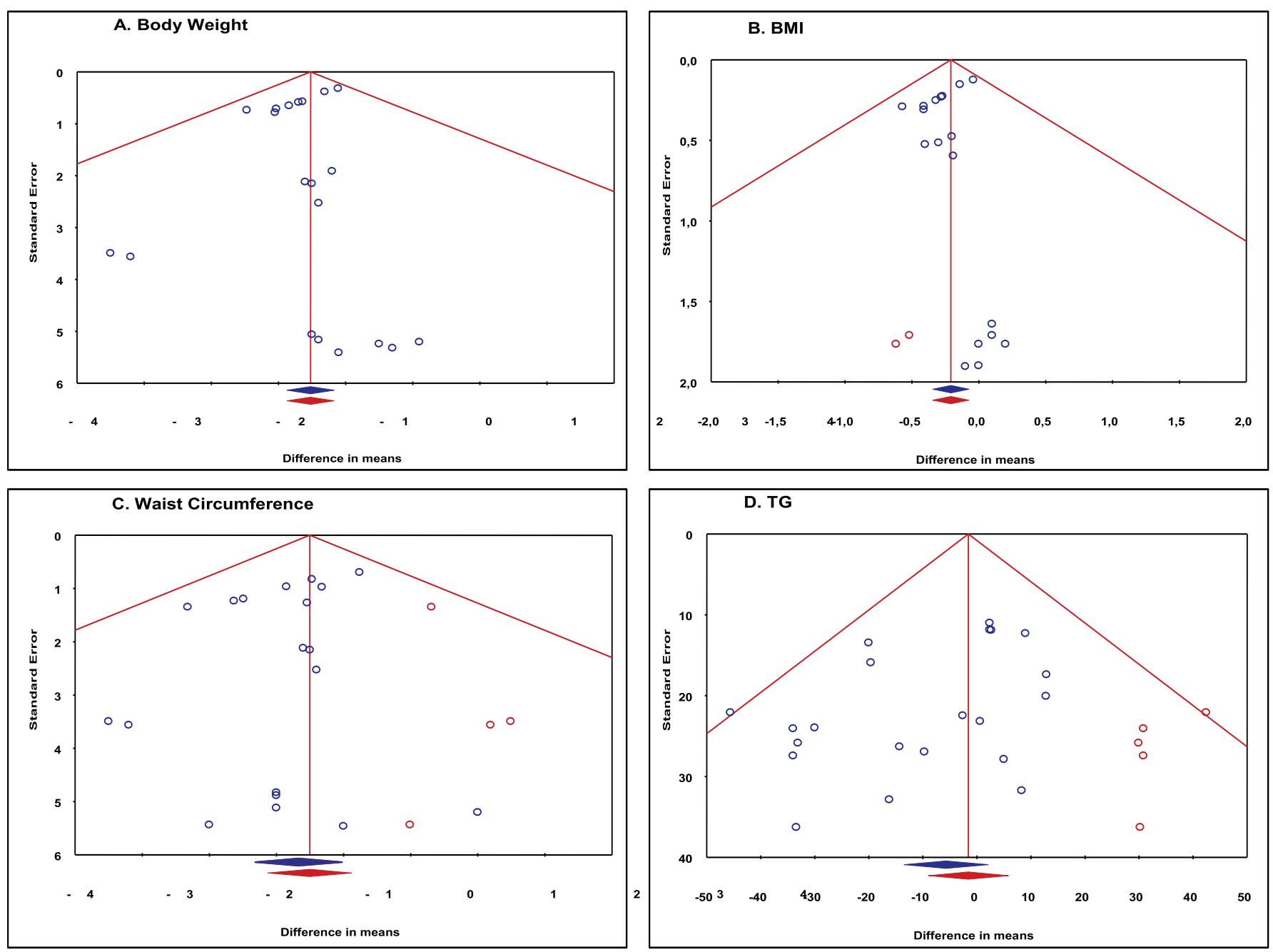

FIGURE 6 - Funnel plots of standard error by difference in means (publication bias tests) of the effects of I. paraguariensis consumption on the outcomes of this meta-analysis. BMI - body mass index; DBP - diastolic blood pressure; HDL - HDLcholesterol; LDL - LDL-cholesterol; SBP - systolic blood pressure; TC - total cholesterol; TG - triglycerides. 

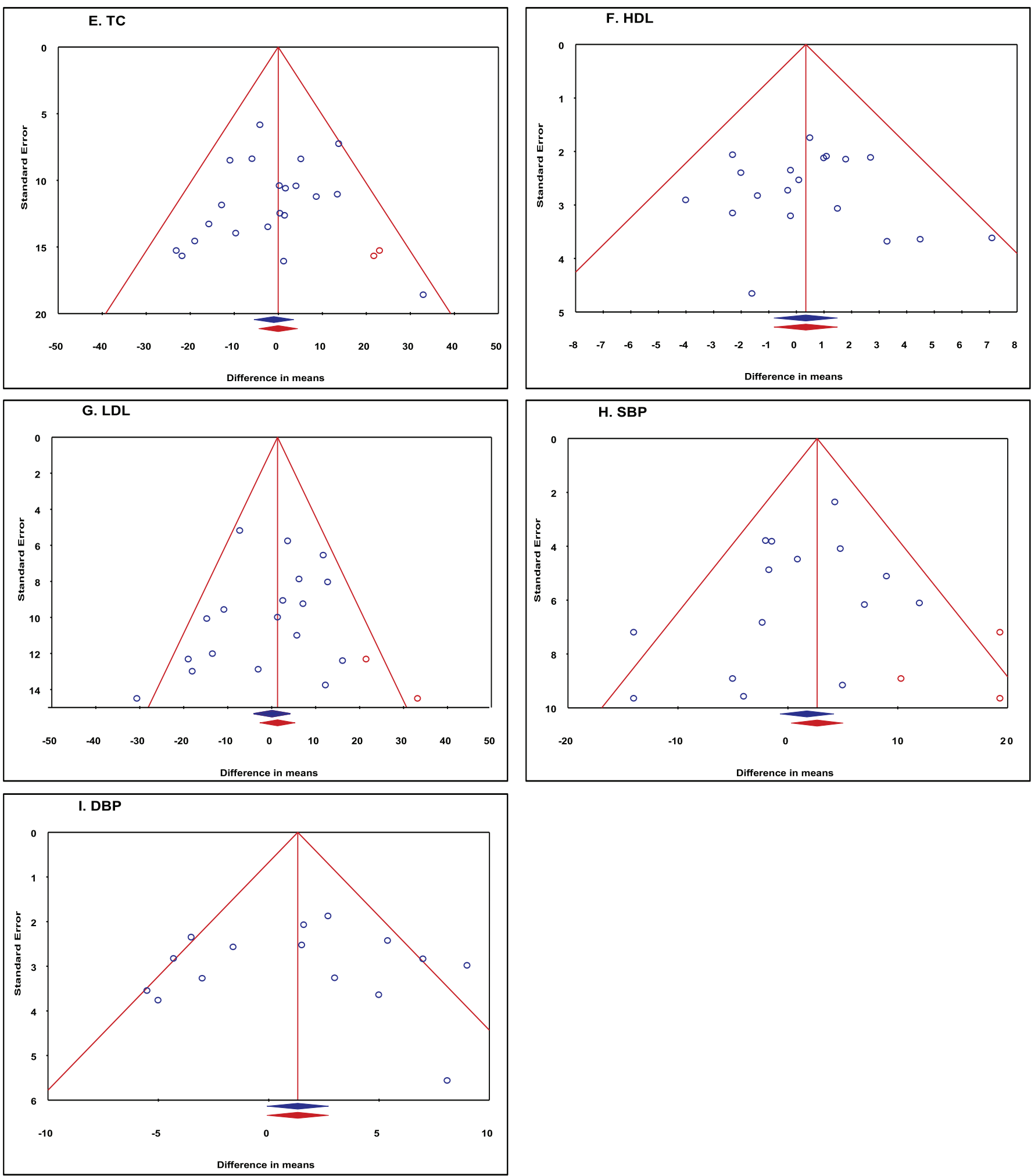

FIGURE 6 (cont.) - Funnel plots of standard error by difference in means (publication bias tests) of the effects of I. paraguariensis consumption on the outcomes of this meta-analysis. BMI - body mass index; DBP - diastolic blood pressure; HDL - HDLcholesterol; LDL - LDL-cholesterol; SBP - systolic blood pressure; TC - total cholesterol; TG - triglycerides. 
I. paraguariensis on lipid profile and blood pressure of patients, together with the evaluation of its consumption by healthy people.

\section{ACKNOWLEDGMENTS}

Ângelo Luís acknowledges the Post-Doc research fellowship within the scope of the protocol signed between Universidade da Beira Interior and bank Santander-Totta with the reference BIPD/ICI-FC-BST-UBI 2016.

\section{REFERENCES}

Boaventura B, Pietro P, Stefanuto A, Klein G, Morais E, Andrade $\mathrm{F}$, et al. Association of mate tea (Ilex paraguariensis) intake and dietary intervention and effects on oxidative stress biomarkers of dyslipidemic subjects. Nutrition. 2012;28(6):657-64.

Borenstein M, Hedges L, Higgins J. Introduction to metaanalysis. Chichester (UK): John Wiley \& Sons; 2009.

Bracesco N, Sanchez A, Contreras V, Menini T, Gugliucci A. Recent advances on Ilex paraguariensis research: Minireview. J Ethnopharmacol. 2011;136(3):378-84.

Cardozo Junior EL, Morand, C. Interest of mate (Ilex paraguariensis A. St.-Hil.) as a new natural functional food to preserve human cardiovascular health - A review. J Funct Foods. 2016;21:440-54.

Duval S, Tweedie R. A non parametric "trim and fill" method of accounting for publication bias in meta-analysis. J Am Statist Assoc. 2000a;95(449):89-98.

Duval S, Tweedie R. Trim and fill: A simple funnel-plot-based method of testing and adjusting for publication bias in metaanalysis. Biometrics. 2000b;56(2):455-63.

Egger M, Davey G, Schneider M, Minder C. Bias in meta-analysis detected by a simple, graphical test. BMJ. 1997;315:629-34.

Gambero A, Ribeiro M. The positive effects of yerba maté (Ilex paraguariensis) in obesity. Nutrients. 2015;7(2):730-50.

Higgins J, Thompson S, Deeks J, Altman D. Measuring inconsistency in meta-analyses. BMJ. 2003;327:557-60.

Higgins J, Altman D, Gotzsche P, Juni P, Moher D, Oxman A, et al. The Cochrane Collaboration's tool for assessing risk of bias in randomised trials. Br Med J. 2011;343:d5928.
Jung J, Hur Y. The Effect of maté extract on body weight and fat reduction in obese women: a randomized placebo-controlled clinical trial. Korean J Obes. 2016;25(4):197-206.

Kim H, Ko J, Storni C, Song H, Cho Y. Effect of green mate in overweight volunteers: A randomized placebo-controlled human study. J Funct Foods. 2012;4(1):287-93.

Kim S, Oh M, Kim M, Chae H, Chae S. Anti-obesity effects of Yerba Mate (Ilex paraguariensis): a randomized, double-blind, placebo-controlled clinical trial. BMC Complement Altern Med. 2015;15:338.

Klein G, Stefanuto A, Boaventura B, Morais E, Cavalcante $\mathrm{L}$, Andrade F, et al. Mate tea (Ilex paraguariensis) Improves glycemic and lipid profiles of Type 2 Diabetes and pre-Diabetes individuals: a pilot study. J Am Coll Nutr. 2011;30(5):320-32.

Light R, Pillemer D. Summing up: The Science of Reviewing Research. Harvard Cambridge, University Press; 1984.

Light R, Singer J, Willett J. The visual presentation and interpretation of meta-analyses, in: Cooper M, Hedges LV. (editors). The Handbook of Research Synthesis. New York: Russell Sage Foundation; 1994.

Moher D, Shamseer L, Clarke M, Ghersi D, Liberati A, Petticrew $\mathrm{M}$, et al. Preferred reporting items for systematic review and meta-analysis protocols (PRISMA-P) 2015 statement. Syst Rev. 2015;4:1.

Morais E, Stefanuto A, Klein G, Boaventura B, Andrade F, Wazlawik E, et al. Consumption of yerba mate (Ilex paraguariensis) improves serum lipid parameters in healthy dyslipidemic subjects and provides an additional LDLcholesterol reduction in individuals on statin therapy. J Agric Food Chem. 2009;57(18):8316-24.

Pontilho P, Teixeira A, Yuan C, Luzia L, Bastos D, Rondó P. Yerba mate (Ilex paraguariensis A. St. Hil) and risk factors for cardiovascular diseases. J Food Nutr Res. 2015;3(3):182-90.

Pontin B. Efeitos da ingestão de chimarrão e chá verde no perfil lipídico e inflamatório de indivíduos com dislipidemia e excesso de peso. [Master's dissertation]. Porto Alegre: Instituto de Cardiologia do Rio Grande do Sul; 2010.

Rein M, Renouf M, Cruz-Hernandez C, Actis-Goretta L, Thakkar S, Pinto M, et al. Bioavailability of bioactive food compounds: a challenging journey to bioefficacy. Br J Clin Pharmacol. 2013;75(3):588-602. 
Rostagno M, Manchón N, D’Arrigo M, Guillamón E, Villares A, García-Lafuente A, et al. Fast and simultaneous determination of phenolic compounds and caffeine in teas, mate, instant coffee, soft drink and energetic drink by high-performance liquid chromatography using a fused-core column. Anal Chim Acta. 2011;685(2):204-11.

Ryan R, Hill S, Prictor M, McKenzie J. Cochrane consumers $\&$ communication review group study quality guide guide for review authors on assessing study. 2013.

Salerno Z. Efeitos do chimarrão (Ilex paraguariensis) na absorção de flavonóides, na concentração dos lípidos e sobre a circulação. Master's dissertation]. Porto Alegre: Universidade Federal do Rio Grande do Sul; 2007.
Thorlund K, Engstrom J, Wetterslev J, Brok J, Imberger G, Gluud C. User Manual for Trial Sequential Analysis (TSA), Copenhagen Trial Unit, Centre for Clinical Intervention Research, Copenhagen, Denmark; 2011.

World Health Organization. [cited 2018 April 25]. Available from: http://www.who.int/news-room/fact-sheets/detail/obesityand-overweight.

Received for publication on $25^{\text {th }}$ September 2017 Accepted for publication on $21^{\text {st }}$ June 2018 